\title{
РЕЗУЛЬТАТЫ ИМИТАЦИОННОГО МОДЕЛИРОВАНИЯ РОБАСТНОЙ СИСТЕМЫ АВТОМАТИЧЕСКОГО РЕГУЛИРОВАНИЯ С НЕЛИНЕЙНЫМ ДИССИПАТИВНЫМ ОБЪЕКТОМ
}

\author{
Павлов А.И. ${ }^{1}$ \\ ${ }^{1}$ Одесская национальная академия пищевых технологий, Одесса
}

Copyright (C) 2014 by author and the journal "Automation technological and business - processes". This work is licensed under the Creative Commons Attribution International License (CC BY). http://creativecommons.org/licenses/by/4.0/

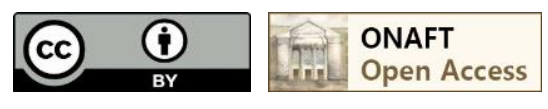

DOI: $10.15673 /$

\begin{abstract}
Аннотация
Показывается возможность реализации в программной среде промышленных контроллеров высокоэффективного автоматического регулятора, использующего простейшую искусственную нейронную сеть.
\end{abstract}

\section{Abstract}

The possibility of realization of the highly effective automatic regulator, using the simplest artificial network in the software of the industrial controllers has been shown

Ключевые слова

Система регулирования, объект, модель объекта, нейронная сеть.

Введение. При решении задач регулирования по-прежнему широко используются линейные ПИДалгоритмы, при том, что технологические регламенты в отношении регулируемых переменных становятся все более жесткими. Обеспечение их выполнения при работе САР с реальным (физическим) объектом в условиях измерения его статических и динамических характеристик все более проблематичным, а нередко и невыполнимым, поскольку грубость таких САР невелика (по А.А. Андронову).

На стадии моделирования (а она является теперь центральной частью этапа проектирования САР) используют обычно инструментальную систему программирования промышленных регулирующих контроллеров, либо пакеты программ Simulink в среде MATLAB. Модель объекта регулирования, как правило, весьма упрощенная (линейная 2-3 порядка с запаздыванием). Статические и динамические характеристики исполнительного устройства подчас и вовсе игнорируются; особенно это относится в варианту использования электрических исполнительных механизмов, а именно они традиционно наиболее часто применяются. В рабочем проекте САР с такими исполнительными устройствами важнейший (с точки зрения динамики реальной САР) параметр исполнительного механизма $\mathrm{T}_{\text {им }}$ (время полного хода при непрерывной его работе), в лучшем случае определяют по номограммам в зависимости от величин параметров объекта регулирования. Как

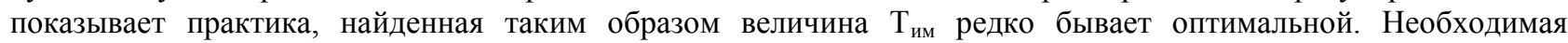

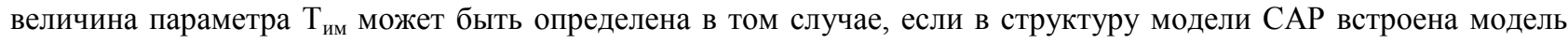
исполнительного устройства.

Но вернемся к стадии моделирования CAP в среде MATLAB. Наличие программ оптимизации параметров регулятора (в его модельном представлении) позволяет сравнительно быстро и, главное, «безболезненно» найти 


\section{$\underline{2}$ АВТОМАТИЧНІ ТА АВТОМАТИЗОВАНІ СИСТЕМИ УПРАВЛІННЯ ТЕХНОЛОГІЧНИМИ ПРОЦЕСАМИ}

численные значения коэффициента передачи $\mathrm{K}_{\mathrm{p}}$, времени изодрома $\mathrm{T}_{\text {из }}$ и времени предварения $\mathrm{T}_{\text {пр }}$ (для ПИДалгоритма) регулятора.

Поскольку часто управляющая часть реальной САР - это регулирующий контроллер с библиотекой программных модулей, в которую входят ПИ-, ПИД-алгоритмы как с непрерывными, так импульсными сигналами управления, то необходимо только «перенести» найденные величины настроек регулятора в программную среду промышленного контроллера. Но и здесь возможны ошибки: во-первых, структура регулирующего алгоритма в контроллере может отличаться от той, которую использовали при моделировании в среде MATLAB; во-вторых, нужно не забыть, что в контроллере вместо параметра «время изодрома» используется параметр «постоянная времени интегрирования $\mathrm{T}_{\text {н }}$ (а они, в общем случае, различны по величине), вместо параметра «время предварения» используется параметр «постоянная времени дифференцирования $\mathrm{T}_{\text {д }}$ (а они также не совпадают).

Чтобы «подстраховаться», разработчик САР может избрать для использования в контроллере ПИ-алгоритм с самонастройкой его основных двух параметров - $\mathrm{K}_{\mathrm{p}}$ и $\mathrm{T}_{\text {и }}$ непосредственно на реальном объекте в процессе работы САР. А это с неизбежностью сопровождается вторжением в технологический процесс и нарушением в той или иной мере его регламента, поскольку необходимо создавать специальные режимы функционирования с довольно большими амплитудами воздействия на объект, а, значит, с существенными отклонениями регулируемого параметра от задания на значительном интервале времени.

В любом случае, окончательно уточняются (корректируются) параметры алгоритма регулирования (особенно это касается ПИД - алгоритма) на стадии наладки регулятора на реально осуществляемом производственном процессе. Но и после этого проблема настройки регулятора, как правило, не исчезла навсегда: по мере «старения» объекта [4] величины его характеристики меняются, и, значит, требуется

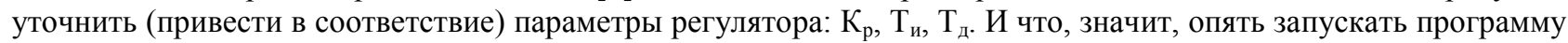
самонастройки регулятора, как это уже было при первом пуске САР в реальную работу? Но ведь еще все, сопричастные к этому, помнят технологические последствия такого эксперимента! Значит, надо идти другим путем, не столь «жестким», то есть использовать более «мягкие» методики - регулярно осуществлять поднастройку параметров регулятора вручную. Это означает необходимость наличия на предприятии высококвалифицированных инженеров-наладчиков (либо периодически «приглашать варягов»). К примеру, на электростанциях число штатных инженеров-наладчиков регуляторов 10 и более человек. Итак, проблема наладки автоматических регуляторов, несмотря на наличие у промышленных контроллеров программ самонастройки, остается весьма актуальной.

Основная часть. Ручная наладка регуляторов и их последующая, повторяющаяся на протяжении всего срока эксплуатации САР, поднастройка становится все более анахронизмом. Другой путь - разработка и все более широкое применение адаптивных систем управления. В числе адаптивных называют обучаемые, самонастраивающиеся, самообучающиеся, самоорганизующиеся системы. Таким образом, в области адаптивных систем управления используется различная терминология, что представляет определенную проблему. «К сожалению, как правило, это термины не имеют однозначного толкования. Это создает благоприятную почву для безудержных фантастических рассуждений» [1].

Сложность реализации адаптивных систем, отягощенная подчас недостаточно высокими динамическими свойствами контура адаптации, нередко становится серьезным препятствием на пути их широкого практического применения.

Альтернативой адаптивному подходу к проблеме «априорной неопределенности» может быть робастный подход [2]. В условиях изменения характеристик внешних воздействий и неконтролируемых параметрических флюктуаций параметров объекта вполне удовлетворительной работы САР можно добиться и без использования алгоритмов адаптации. Для этого необходимо синтезировать систему управления с ее постоянными параметрами таким образом, чтобы при действии возмущающих факторов качество работы САР не опускалось ниже определенного допустимого технологическим регламентом уровня. Такие системы регулирования, альтернативные адаптивным, получившие наименование робастных, конечно не могут соперничать по качеству управления с адаптивными. Но в тех случаях, когда не требуется очень высокое качество регулирования, существенное преимущество робастных систем перед адаптивными, состоящее в простоте их реализации, неоспоримо. Именно поэтому проектирование и более широкое применение робастных систем управления является актуальным.

Синтез робастных САР возможен на основе разнообразных идей, методов и технологий. В частности, эффективные решения могут быть получены посредством применения непрерывной (нечеткой) логики либо искусственных нейронных сетей. Основной задачей данной статьи, которую ставил перед собой автор, было намерение показать, что САР приобретает робастные свойства при управлении параметром (например, 


\section{$\underline{2}$ АВТОМАТИЧНІ ТА АВТОМАТИЗОВАНІ СИСТЕМИ УПРАВЛІННЯ ТЕХНОЛОГІЧНИМИ ПРОЦЕСАМИ}

температурой) нелинейного объекта на основе использования возможностей, представляемых нейронными технологиями.

Исходя из принципа простоты, количество входных сигналов регулятора ограничено четырьмя: сигналом задания, сигналом датчика контролируемого возмущения, действующего по каналу управления (конкретно отклонение давления пара от номинального значения) и динамическая ошибка регулирования.

Функция активации - линейная с двумя ограничениями: снизу и сверху. Предусмотрено нормирование сигнала управления (соответствие его диапазону $0 . .100 \%$ ).

Имитационная модель исполнительного механизма соответствует пневматическому мембранному, оснащенного позиционером с ПД-алгоритмом, а регулирующего органа - с равнопроцентной пропускной характеристикой.

Результаты имитационного моделирования представлены на рис. 1 и 2.

На рис. 1 показано влияние на динамику регулируемой переменной уменьшения величины коэффициента передачи объекта $\mathrm{K}_{\mathrm{o}}(\mathrm{t})$.

Но обычно в «связке» с $\mathrm{K}_{\mathrm{o}}(\mathrm{t})$ находятся, меняясь в процессе «старения» объекта, и другие его параметры: $\mathrm{T}_{1}$, $\mathrm{T}_{2}$, и $\tau$; при этом $\tau / \mathrm{T}$ обычно возрастает. Естественно возникает вопрос о влиянии изменений всей совокупности параметров объекта на работу САР.

Динамика регулируемой переменной при существенно изменившихся их величинах показана на рис. 2 (коэффициент передачи технологического аппарата снижен в 4 раза, запаздывание увеличено в 4 раза, постоянные времени $\mathrm{T}_{1}$ и $\mathrm{T}_{2}$ увеличены втрое).

Для обеспечения инвариантности регулируемой переменной от больших изменений параметров объекта потребовало введения в управляющую часть системы корректирующих цепей, сравнительно простых по своей структуре. Однако их необходимость вполне обусловлена: усложнив модель объекта, по необходимости (для обеспечения «равновесия») требуется усложнить и управляющую часть.

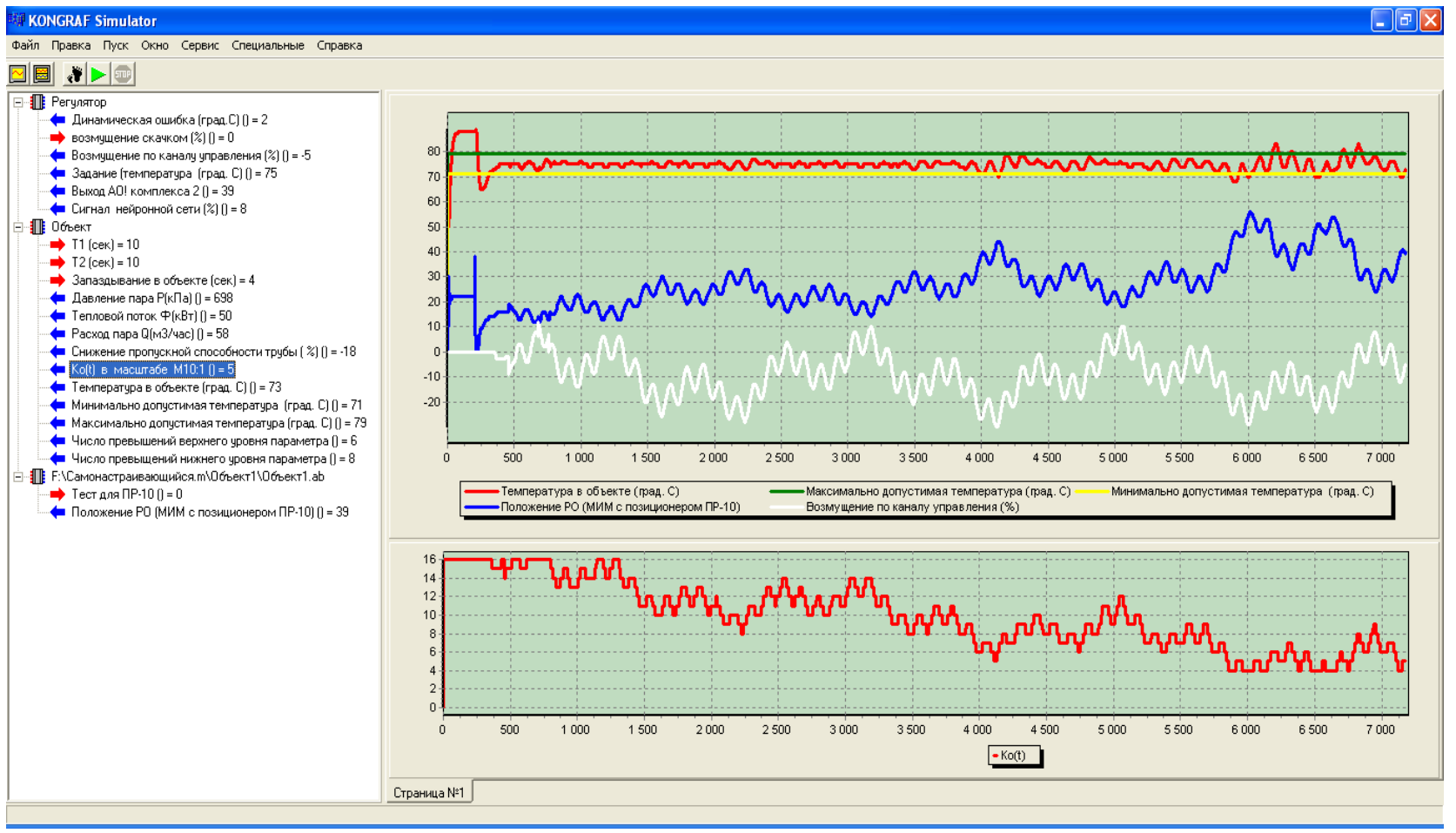

Рис. 1 - Результат моделирования САР $\left(\mathrm{K}_{\mathrm{o}}(\mathrm{t})=1,6 \ldots 0,4 ; \mathrm{T}_{1}=\mathrm{T}_{2}=10 \mathrm{c}\right.$; запаздывание $\left.\tau=4 \mathrm{c}\right)$ 


\section{$\underline{2}$ АВТОМАТИЧНІ ТА АВТОМАТИЗОВАНІ СИСТЕМИ УПРАВЛІННЯ ТЕХНОЛОГІЧНИМИ ПРОЦЕСАМИ}

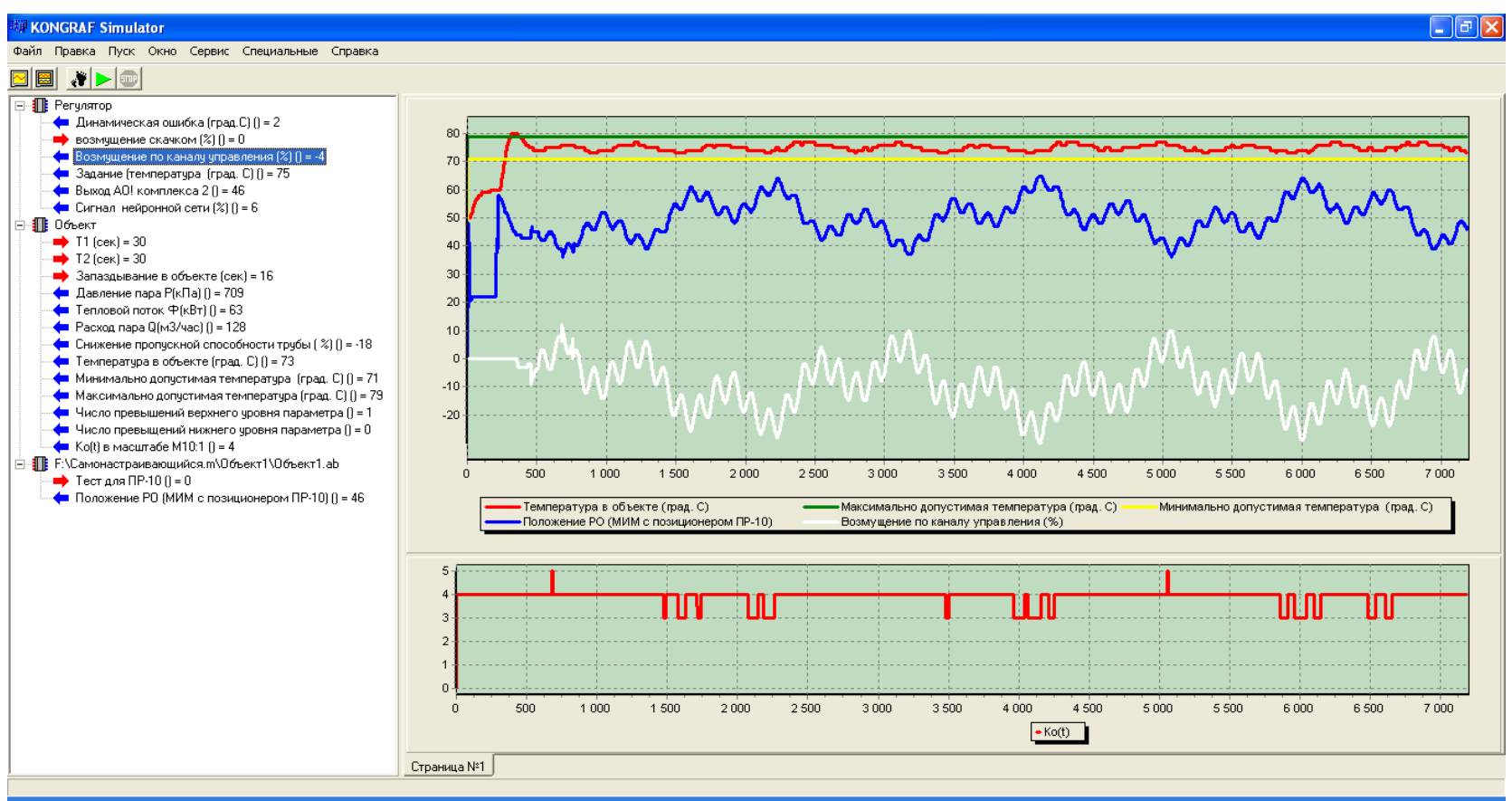

Рис. 2 - Результат моделирования САР при существенно измененных параметрах объекта $\left(\mathrm{K}_{\mathrm{o}}(\mathrm{t})=0,4 ; \mathrm{T}_{1}=\mathrm{T}_{2}=30 \mathrm{c}\right.$; запаздывание $\left.\tau=16 \mathrm{c}\right)$

Выводы. Рассмотрение процессов преобразования и взаимодействия параметров, характеризующих работу всех частей САР, позволяет существенно повысить адекватность ее модели физическим процессам в реальной системе.

В условиях значительных параметрических возмущений, действующих на объект регулирования, использование нейрорегуляторов позволяет успешно компенсировать их совокупность, однако делает необходимым введение корректирующих цепей.

Управляющая часть САР легко реализуется в программной среде промышленных регулирующих контроллеров, оснащенных развитыми библиотеками программ (например, MC5, MC8 комплекса KONTAR; P-130ISA).

Сопоставление динамики регулируемой переменной на рис. 1 и 2 позволяет утверждать, что качество управления не только не снизилось, как можно было ожидать, но даже несколько повысилось. Но это вполне закономерно, поскольку во втором случае (рис. 2) величина $\mathrm{K}_{\mathrm{o}}(\mathrm{t})$ менялась не существенно и реже.

\section{Литература}

1. Цыпкин Я. 3. Адаптация и обучение в автоматических системах / Я. 3. Цыпки // М. : Наука, 1968. - 400 $\mathrm{c}$;

2. Бесекерский В. А. Робастные системы автоматического управления / В. А. Бесекерский, А. В. Небылов // М.: Наука, 1983. - 240 с;

3. Павлов А. И. Повышение адекватности имитационных моделей нелинейных дисеппативных объектов / А. И. Павлов // Автоматизация технологических и бизнес-процессов. - Одесса : ОНАПТ, 2014. - № 17. C. 4-7;

4. Воинова С. А. Особенности управления техническими объектами на траектории расходования расчетного ресурса / С. А. Воинова // Автоматизация технологических и бизнес-процессов. - Одесса : ОНАПТ, 2010. - № 1. - С. 10-13;

5. Павлов А. И. Повышение эффективности управляющих воздействий автоматических регуляторов / А. И. Павлов // Автоматизация технологических и бизнес-процессов». - Одесса : ОНАПТ, 2011. - № 5, 6. - С. $31-37$. 